\title{
Erratum: Suppressing decoherence in quantum plasmonic systems by the spectral-hole-burning effect [Phys. Rev. A 103, 053517 (2021)]
}

Jia-Bin You $\odot$, Xiao Xiong, Ping Bai, Zhang-Kai Zhou, Wan-Li Yang, Ching Eng Png, Leong Chuan Kwek, and Lin Wu (Received 7 July 2021; published 19 July 2021)

DOI: 10.1103/PhysRevA.104.019901

The text "More recently... norm of a state" in Appendix B 2 of our paper [except Eqs. (B12)-(B15)] was largely reproduced from the work of Secular et al. [1] for limited purposes of the description of theoretical methods. However, although that work was cited, we overlooked including quotation marks or a clear statement of attribution to indicate that much of the area in question was taken directly from that work. We apologize for the oversight.

[1] P. Secular, N. Gourianov, M. Lubasch, S. Dolgov, S. R. Clark, and D. Jaksch, Phys. Rev. B 101, 235123 (2020). 